\title{
The Problem of Overlapping Formation Times: A (nearly) complete result for QCD
}

\author{
Peter Arnold \\ Department of Physics, University of Virginia, \\ Charlottesville, Virginia 22904-4714, U.S.A \\ E-mail: parnoldevirginia.edu

\section{Tyler Gorda} \\ Department of Physics, University of Virginia, \\ Charlottesville, Virginia 22904-4714, U.S.A \\ E-mail: tyler.gorda@physik.tu-darmstadt.de
}

\section{Shahin lqbal*}

Institute of Particle Physics, Central China Normal University

Wuhan, China

E-mail: smi6ndevirginia.edu

\begin{abstract}
The splitting processes of bremsstrahlung and pair-production in a medium are coherent over large distances in the high energy limit leading to a suppression known as the Landau-PomeranchukMigdal (LPM) effect. Avoiding soft-emission approximations and working in the large-Nc limit, we consider corrections to the LPM effect from cases where the coherence lengths of two consecutive splittings overlap. In this work, we present (i) complete results for the case of two overlapping gluon splittings (e.g. $g \rightarrow g g \rightarrow g g g$ and virtual corrections to single splitting $g \rightarrow g g^{*} \rightarrow g g g^{*} \rightarrow g g$ ) and (ii) confirm that earlier leading-log results for these effects are reproduced by our more-complete results in the appropriate soft limit. We also discuss how to combine the effects of overlapping real double splitting with the corresponding virtual corrections to single splitting in order to calculate IR-safe quantities such as in-medium energy loss.
\end{abstract}

HardProbes 2020

1-6 June 2020

Austin, Texas

${ }^{*}$ Speaker. 


\section{Introduction}

High energy particles passing through matter primarily lose energy by showering, i.e. splitting up through bremsstrahlung and pair-production. At high energies, the quantum-mechanical "duration" of the splitting process, called the formation time, exceeds the mean free time between collisions in the medium. This leads to significant reduction in the splitting rate and is known as the Landau-Pomeranchuk-Migdal (LPM) effect [1,2,3]. A longstanding problem in field theory had been to calculate this effect in cases when the formation times of two consecutive splitting processes overlap. This problem has been analyzed by previous authors in the soft emission limitwhere the energy of the radiated particles is much smaller than the parent - and found important leading-log corrections to $\hat{q}[11,12,13]$. In our previous work, together with Chang and Rase, avoiding soft emission approximations we have calculated the $O\left(\alpha_{s}\right)$ corrections to the rate of real double gluon bremsstrahlung $g \rightarrow g g \rightarrow g g g[4,5,6,7]$ and in-medium virtual corrections for large-Nf QED where Nf is the number of electron flavors[8, 9]. In particular, we found that the overlap effects from real double bremsstrahlung would produce power-law IR divergences in calculations of in-medium energy loss. However, in order to calculate overlapping formation time effects on IR safe quantities such as in-medium energy loss, the corresponding in-medium virtual corrections to single splitting $g \rightarrow g g$ must also be calculated. In the current work, we will focus on calculating in-medium virtual corrections to single gluon bremsstrahlung and how to combine them with our previous calculations for calculating IR safe quantities. In our analysis of virtual corrections, we also verify that the known UV renormalization of the QCD coupling constant is correctly reproduced. Similar to the assumptions in our previous work on this topic, we will use the large-Nc limit of QCD and will work in the often used $\hat{q}$ approximation (also called the multiple scattering approximation). We will also assume that the background medium is homogeneous and large compared to the typical formation length. Details of the calculation and results presented here have been published in our recent paper [10].

\section{Overview of the calculation}

To qualitatively understand the LPM effect consider the leading order LPM effect calculation for an electron radiating a bremsstrahlung photon as a result of collisions in the medium. The radiated photon cannot resolve details smaller than its wavelength, which creates an uncertainty about the exact time and place of the emission process. The extent of this uncertainty is called the formation length (formation time) and we have depicted it as the blue shaded region in Fig. 1. Because of this ambiguity, when the wavelength of the radiated photon becomes larger than the mean free path between collisions, a bremsstrahlung resulting from a single scattering becomes indistinguishable from a bremsstrahlung resulting from multiple small angle scatterings in the medium, as shown in the Fig. 1. The observed bremsstrahlung rate is then smaller than what one would have naively expected.

In terms of Feynman diagrams, the LPM effect results from the quantum interference of splitting amplitudes from different scattering centers as shown in Fig. 2. The upper blue part of the diagram represents the amplitude for an in-medium gluon bremsstrahlung, and the red represents the conjugate amplitude part of an interference contribution to the rate. For diagrammatic con- 


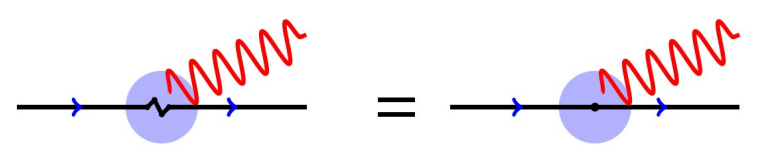

Figure 1: Bremsstrahlung from an electron scattering in the medium. The diagram on the left depicts bremsstrahlung from a series of scatterings with the medium which becomes indistinguishable from bremsstrahlung from a single scattering as shown in the diagram on the right.

venience, only the high energy particles are shown explicitly and the implicit interactions with particles in the medium have been omitted in Fig. 2. For calculations like these it is helpful to reinterpret this time-ordered interference contribution as a single process, with a 3-particle in-medium evolution sandwiched between splitting matrix elements at times $t$ and $\bar{t}$, as shown in the diagram on the right in Fig. 2. Splitting matrix elements are calculated from QCD Feynman rules and the medium averaged time evolution between splitting vertices is governed by an effective nonHermitian Hamiltonian. Finally, if one chooses to work in the often used $\hat{q}$ approximation, the effective Hamiltonian becomes that of a Harmonic Oscillator with complex valued frequencies. The leading order differential splitting rate for $g \rightarrow g g$ in a homogeneous, thick and static medium

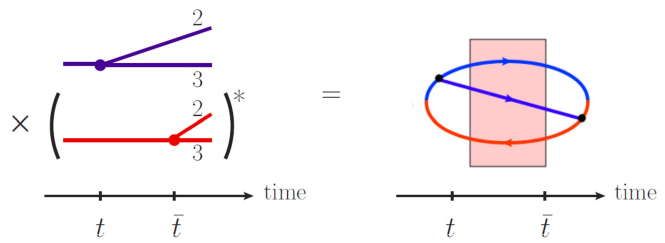

Figure 2: Schematic interference diagram for a leading order in-medium single splitting process. We have only shown the high energy particles and omitted their many interactions with the medium for diagrammatic convenience.

at leading order in $\alpha_{s}$ is then given by

$$
\left[\frac{d \Gamma}{d x}\right]_{L O}=2 \operatorname{Re}\left[\frac{d \Gamma}{d x}\right]_{x \bar{x}}=\frac{\alpha_{s}}{\pi} P_{g \rightarrow g, g}(x) \operatorname{Re}\left(i \Omega_{0}\right) .
$$


Here, $E$ is the energy of the initial particle, $x$ is the energy fraction of the bremmstrahlung gluon, $P_{g \rightarrow g, g}(x)$ is the relevant DGLAP splitting function and

$$
\Omega_{0}=\sqrt{-i \frac{\hat{q}}{2 E}\left(-1+\frac{1}{x}+\frac{1}{1-x}\right)} .
$$

Now, let $x$ and $y$ be the energy fractions of two sequentially radiated gluons with overlapping formation times. Understanding the LPM effect in such cases had been a longstanding problem in field theory. In our previous work, together with Chang and Rase, we developed field theory formalism to calculate overlapping formation time corrections and calculated overlap effects in the case of real double gluon bremsstrahlung $g \rightarrow$ ggg. However, we found that the effect of overlapping formation times is in general to enhance the double splitting rate unless one of the radiated gluons is much softer than the other two, e.g. in the limit $y \ll x \ll 1$ in which case the correction to the rate is approximately,

$$
\Delta\left[\frac{d \Gamma}{d x d y}\right]_{N L O}^{g \rightarrow g g g} \sim-\frac{\alpha_{s}}{x y^{3 / 2}} \sqrt{\frac{\hat{q}}{E}} .
$$

Clearly, this would produce power-law IR divergences in calculations of in-medium energy loss. However, a complete calculation of in-medium energy loss at $O\left(\alpha_{s}\right)$ must also include the corresponding $O\left(\alpha_{s}\right)$ corrections to leading order single splitting as shown in Fig. 3. In the present work, we present our results for all such in-medium one loop virtual corrections to single bremsstrahlung, again working in the $\hat{q}$ approximation and using the large-Nc limit of QCD. It should however be noted that the present work does not include calculation of contributions from processes involving the 4-gluon vertex of QCD, as well as those that involve longitudinal polarizations of intermediate state gauge bosons, and is the reason for "nearly" in the title. We have left that calculation for later work.

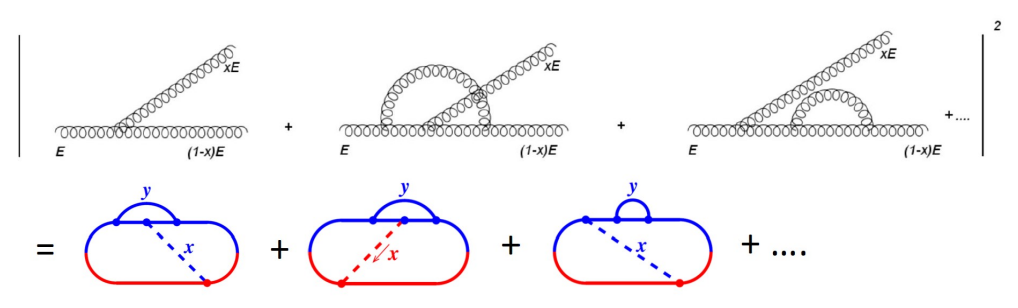

Figure 3: One loop virtual diagrams contributing to the single gluon bremsstrahlung. 


\section{Results}

Individually, each time-ordered overlapping virtual diagram such as shown in Fig. 3 is UV divergent. This is also true for all overlapping real double bremsstrahlung diagrams considered in our previous work [4, 5], but all such UV divergences cancel when all possible time orderings are added together. However, it turns out that such UV divergences from one-loop virtual diagrams do not all cancel and require highly non-trivial regularization. Using dimensional regularization we find that the total UV divergent contribution from all of the virtual diagrams correctly reproduces the known UV renormalization of the QCD coupling constant. Specifically, using MS-bar renormalization we find that full next-to-leading order correction from all one-loop virtual diagrams can be expressed as

$$
\left[\frac{d \Gamma}{d x}\right]_{g \rightarrow g g}^{N L O}=-\beta_{0} \alpha_{s} \operatorname{Re}\left(\left[\frac{d \Gamma}{d x}\right]_{x \bar{x}}\left[\ln \left(\frac{\mu^{2}}{\Omega_{0} E}\right)+\ln \left(\frac{x(1-x)}{4}\right)+\gamma_{E}\right]\right)+\left[\Delta \frac{d \Gamma}{d x}\right]_{g \rightarrow g g}^{\overline{N L O}},
$$

with $\varepsilon \rightarrow 0$. Here $\beta_{0}=-\frac{11}{6 \pi}$ is the one-loop QCD beta function, $\mu$ is the renormalization scale and $\left[\Delta \frac{d \Gamma}{d x}\right]_{g \rightarrow g g}^{\overline{N L O}}$ contains complicated integrals that can be performed numerically.

As mentioned earlier, when calculating $O\left(\alpha_{s}\right)$ corrections to energy loss, contributions from the real $g \rightarrow g g g$ must be supplemented by the corresponding $O\left(\alpha_{s}\right)$ virtual corrections to single splitting $g \rightarrow g g$. That is, the relevant splitting rate for calculating NLO $O\left(\alpha_{s}\right)$ corrections to inmedium energy loss can be written as

$$
\left[\frac{d \Gamma}{d x}\right]^{n e t}=\left[\frac{d \Gamma}{d x}\right]_{g \rightarrow g g}^{L O}+\left[\frac{d \Gamma}{d x}\right]_{g \rightarrow g g}^{N L O}+\left[\Delta \frac{d \Gamma}{d x}\right]_{g \rightarrow g g g}^{N L O},
$$

We find that all power-law IR divergences are cancelled between real and virtual NLO contributions, leaving behind the known double-log and a previously unknown single-log IR divergence, i.e.

$$
\left[\frac{d \Gamma}{d x}\right]^{n e t} \sim\left(1+\frac{C_{A} \alpha_{s}}{8 \pi}\left(\ln \delta^{2}+s(x) \ln \delta\right)\right)\left[\frac{d \Gamma}{d x}\right]_{g \rightarrow g g}^{L O}
$$

where $\delta$ is our IR cutoff on the value of radiated gluon's energy fraction $y$. For details of the choice of IR cutoff and a discussion of the behavior of the single-log coefficient $s(x)$, we would refer the readers to [10].

\section{Acknowledgments}

This work was supported, in part, by the U.S. Department of Energy under Grant No. DE-SC0007984 (Arnold and Gorda) and the National Natural Science Foundation of China under Grant Nos. 11935007, 11221504 and 11890714 (Iqbal).

\section{References}

[1] L.D. Landau and I. Pomeranchuk, Limits of applicability of the theory of bremsstrahlung electrons and pair production at high-energies (in Russian), Dokl. Akad. Nauk Ser. Fiz. 92 (1953) 535. 
[2] L.D. Landau and I. Pomeranchuk, Electron cascade process at very high energies (in Russian), Dokl. Akad. Nauk Ser. Fiz. 92 (1953) 735.

[3] A.B. Migdal, Bremsstrahlung and pair production in condensed media at high-energies, Phys. Rev. 103 (1956) 1811.

[4] P. Arnold and S. Iqbal, "The LPM effect in sequential bremsstrahlung", JHEP 04 (2015) 070 [arXiv:1501.04964 [hep-ph]].

[5] P. Arnold, H.-C. Chang and S. Iqbal, "The LPM effect in sequential bremsstrahlung 2: factorization", JHEP 09 (2016) 078 [arXiv:1605.07624[hep-ph]].

[6] P. Arnold, H.-C. Chang and S. Iqbal, "The LPM effect in sequential bremsstrahlung: dimensional regularization", JHEP 10 (2016) 100 [arXiv:1606.08853[hep-ph]].

[7] P. Arnold, H.C. Chang and S. Iqbal "The LPM effect in sequential bremsstrahlung: 4-gluon vertices", JHEP 10 (2016) 124 [arXiv:1608.05718[hep-ph]].

[8] P. Arnold and S. Iqbal "In-medium loop corrections and longitudinally polarized gauge bosons in high energy showers", JHEP 12 (2018) 120 [arXiv:1806.08796 [hep-ph]].

[9] P. Arnold, S. Iqbal and Tanner Rase "Strong-vs. weak coupling pictures of jet quenching: A dry run using QED”, JHEP 05 (2019) 004 [arxiv: 1810.06578[hep-ph]]

[10] P.Arnold, T. Gorda and S. Iqbal "The LPM effect in sequential bremsstrahlung: nearly complete results for QCD", arXiv:2007.15018 [hep-ph].

[11] B. Wu, "Radiative energy loss and radiative p-broadening of high-energy partons in QCD matter," JHEP 1412, 081 (2014) [arXiv:1408.5459 [hep-ph]].

[12] J. P. Blaizot and Y. Mehtar-Tani, "Renormalization of the jet-quenching parameter," Nucl.Phys. A 929, 202 (2014) [arXiv:1403.2323 [hep-ph]].

[13] E. Iancu, "The non-linear evolution of jet quenching," JHEP 1410, 95 (2014) [arXiv:1403.1996 [hep-ph]]. 